Laszlo A. Vaskovics*

\title{
30 Jahre Zeitschrift für Familienforschung/Journal of Family Research - ein Rückblick
}

\section{Vorwort}

Obwohl ich als ehemaliger geschäftsführender Herausgeber direkt in die Entwicklung der Zeitschrift involviert war, versuche ich aus der nötigen kritischen Distanz rückblickend über die Entwicklung der Zeitschrift im Laufe der vergangenen drei Jahrzehnte ein objektives Bild zu skizzieren. Zunächst beschreibe ich die Phasen der Entwicklung, ihre Zäsuren und Charakteristika. Anschließend versuche ich den „Output“ der Zeitschrift darzustellen und ziehe abschließend ein, von persönlichen Wertungen sicherlich nicht ganz freies, Resümee.

\section{Etappen und Phasen der Entwicklung der ZFF/JFR}

\subsection{Institutseigene Zeitschrift des Staatsinstituts für Frühpädagogik und Familienforschung in München (Jahrgänge 1 bis 5, 1989-1993)}

Der 1. Jahrgang der Zeitschrift für Familienforschung ist im Jahre 1989 erschienen. Sie richtete sich in ihrer ursprünglichen Gestaltung als institutseigenes Publikationsorgan des Staatsinstituts für Frühpädagogik und Familienforschung in München primär an das wissenschaftliche und berufsorientierte Fachpublikum in den deutschsprachigen Ländern Europas. Als Adressaten wurden (im Editorial des 1. Heftes) Wissenschaftler, die sich mit familienbezogenen Forschungsfragen befassen, benannt, aber auch Praktiker, die sich von Berufs wegen um Familien und familienbezogene Probleme kümmern und sich über aktuelle Ergebnisse wissenschaftlicher Familienforschung informieren wollten (z.B. Ärzte, Therapeuten, Fachpersonal in sozialen Einrichtungen). Veröffentlicht wurden in dieser

* Unter Mitarbeit von Kurt P. Bierschock. 
Entwicklungsphase, neben wissenschaftlichen Beiträgen von Psychologen, Pädagogen, Soziologen und Demografen, institutseigene Berichte, aktuelle Kurzmitteilungen über Tagungen und Kongresse, Rezensionen von Mitarbeitern des Instituts sowie eine aktuelle Liste von Neuerscheinungen im Bereich der Familienforschung. In der Zeitschrift sollten in erster Linie wissenschaftliche Originalarbeiten veröffentlicht werden; sie hat aber auch eine Plattform zur Verfügung gestellt, auf der auch die Umsetzung von aktuellen Forschungsergebnissen unter Einbezug von Vertretern der Praxis beschrieben und diskutiert werden sollten.

Die Redaktion der Zeitschrift befand sich im Staatsinstitut für Frühpädagogik und Familienforschung in München. Geschäftsführender Herausgeber war von 1989 bis 1993 Wassilios Fthenakis, der damalige Leiter des Institutes.

\subsection{Institutseigene Zeitschrift des Staatsinstituts für Familienforschung an der Universität Bamberg (Jahrgänge 6 bis 9, 1994-1997)}

Nach einer längeren Vorbereitungszeit wurde die Zeitschrift für Familienforschung nach dessen Gründung im Jahre 1994 vom Staatsinstitut für Familienforschung an der Universität Bamberg (ifb) übernommen und als wissenschaftliches Publikationsorgan herausgegeben - mit einem neuen Status versehen, nach einer neuen inhaltlichen Konzeption strukturiert und durch neue Herausgeber verantwortet. Das ifb hat die Zeitschrift personell und durch apparative Ausstattungen unterstützt, es übernahm auch die Redaktion der Zeitschrift. Die Herausgeberschaft wurde von fünf Wissenschaftlern mit unterschiedlichen Forschungsschwerpunkten übernommen. Die personelle Zusammensetzung des Herausgebergremiums sollte die wissenschaftliche Unabhängigkeit der Zeitschrift und das wissenschaftliche Niveau gewährleisten. Zugleich sollten die neuen Herausgeber der Zeitschrift ein neues wissenschaftliches Profil geben.

Die Herausgeberschaft haben in dieser Phase Wolfgang Glatzer (Universität Frankfurt am Main), François Höpflinger (Universität Zürich), Ilona Ostner (Universität Göttingen), Norbert F. Schneider (Universität Mainz) und geschäftsführend Laszlo A. Vaskovics (Universität Bamberg) übernommen. Gemäß der Geschäftsordnung ist der Leiter des ifb zugleich geschäftsführender Herausgeber der Zeitschrift.

Gemäß der neuen Konzeption, die die Herausgeber auf ihrer konstituierenden Sitzung am 22.11.1997 beschlossen hatten, soll die Zeitschrift die interdisziplinäre Kommunikation und Diskussion durch die Veröffentlichung von Beiträgen zur Familien- und Haushaltsforschung fördern. Dies unter Einbeziehung verschiedener Fachdisziplinen - insbesondere der Familiensoziologie, der Familiendemografie, der Familienpsychologie, Familienpädagogik, Haushaltswissenschaft und der historischen Familienforschung. Vorzugsweise sollen Beiträge veröffentlicht werden, die die Ergebnisse theoriegeleiteter empirischer Forschung beschreiben und zur Diskussion stellen oder aber den gegenwärtigen Forschungsstand zu ausgewählten Gegenstandsbereichen der Familienforschung kritisch resümieren. Anvisiert waren auch Beiträge, die über die empirische Beschreibung und theoretische Erklärung hinausgehend, Ansätze zur praktischen Anwendung der Forschungsergebnisse bieten. Insbesondere sollten Manuskripte eingeworben wurden, die über die Beschreibung und sozialwissenschaftliche Erklärung von Daseinsformen und Wandel deutscher Familien und Haushalte hinaus, ländervergleichende Analysen enthalten. 
Die Struktur der Zeitschrift wurde zwar auch nach Übernahme der Trägerschaft und Herausgabe der Zeitschrift durch das neugegründete ifb vorübergehend beibehalten, doch wurden bereits Überlegungen auch zum künftigen Status der Zeitschrift angestellt.

Neben wissenschaftlichen Abhandlungen sollten unter der Rubrik „Forum“ u.a. kurze Diskussionsbeiträge veröffentlicht werden, die den aktuellen Stand der Familienforschung und den Stand der Familienentwicklung sowie familienbezogene politische Maßnahmen beschreiben und diskutieren. Es wurde auch die Veröffentlichung von Literaturberichten (Sammelbesprechungen) zu ausgewählten Themenbereichen der Familienforschung angestrebt, um die Leserinen und Leser der $Z f F$ über den aktuellen Stand der internationalen Forschungsliteratur in kritisch würdigender Weise zu informieren. Auch über die Ergebnisse ausgewählter Tagungen und Kongresse, die im Bereich der Familienforschung stattfinden, sollte unter der Rubrik „Nachrichten" kritisch würdigend berichtet werden. Die Zeitschrift sollte dreimal im Jahr - im April, September und Dezember - im Umfang von 100 bis 130 Druckseiten pro Heft erscheinen.

Zugleich verfolgten die Herausgeber das Ziel, in unregelmäßigen Abständen Sonderhefte in Buchform zu ausgewählten, aktuellen Schwerpunktthemen zu veröffentlichen.

\subsection{Verlagszeitschrift ZfF/JFR (ab Jahrgang 10, ab 1998)}

Die Zeitschrift sollte schon 1995/96 in einem Verlag erscheinen, die Realisierung der Pläne hat sich z.T. aus rechtlichen und auch finanziellen Gründen als schwierig und langwierig erwiesen. Ein großzügiges Angebot des Verlages Leske + Budrich hat dann im Jahre 1998 die mit Rückschlägen belastete Suchphase beendet und die Weiterführung der Zeitschrift als unabhängige, auf dem Buchmarkt allgemein zugängliche, wissenschaftliche Fachzeitschrift ermöglicht.

Die Jahrgänge 10-15 (1998-2003) sind bei Leske + Budrich, die Jahrgänge 16 bis 17 (2004-2005) beim Verlag für Sozialwissenschaften erschienen. Seit dem Jahrgang 18 (2006) erscheint die Zeitschrift beim Verlag Barbara Budrich/Barbara Budrich Publishers.

Die im Jahre 1998 durchgeführte Umwandlung der bis dahin institutseigenen Zeitschrift zu einer einer Verlagszeitschrift hat sich für die künftige Entwicklung als in mehrfacher Hinsicht entscheidend erwiesen. Aus diesem Anlass wurde die inhaltliche und strukturelle Konzeption der $Z f F$ erneut verändert. Der Neukonzipierung als Verlagszeitschrift ging eine durchaus kontrovers geführte Diskussion über die Notwendigkeit einer wissenschaftlichen Zeitschrift für Familienforschung voraus. Die wichtigsten Gegenargumente waren durchaus gewichtig. Manche vertraten die Auffassung, dass wegen des die Familie betreffenden Wandels - etwa: Pluralisierung der Lebensformen, De-Institutionalisierung der Familie, rasche Verbreitung nichtehelicher Lebensgemeinschaften - sich der Gegenstandsbereich der Familienforschung allmählich auflöst oder so stark verändert, dass dieser mit den bisherigen Methoden der (sozialwissenschaftlichen) Familienforschung nicht mehr zu „greifen“ sei. Andere meinten, dass in der ohnehin hochgradig ausdifferenzierten „Zeitschriftenlandschaft“ eine weitere Zeitschrift - egal mit welcher inhaltlichen Ausrichtung - schlicht überflüssig ist, und wieder andere wandten ein, dass eine solche Zeitschrift finanziell nicht überleben kann.

Doch die Entwicklung des Interesses an Familienfragen in den „90er Jahren“ hat eher die Einschätzung bestätigt, dass der Gegenstandsbereich der Familie für verschiedene 
Fachdisziplinen (insbesondere Soziologie, Psychologie, Pädagogik, Demografie) seine Bedeutsamkeit durchaus nicht verloren hat, sondern - im Gegenteil - die Bedeutung der Familie sowohl in der Öffentlichkeit wie auch in der wissenschaftlichen Forschung zugenommen hat und weiterhin zunehmen wird. Darüber hinaus hat sich bewahrheitet, dass sich die Fragen der Familienforschung immer mehr disziplinübergreifend stellen und interdisziplinär zu beantworten sind. Die Familienforschung hat in den 1980er und 1990er Jahren einen erfreulichen Aufschwung genommen und zugleich auch zunehmendes Interesse in der Öffentlichkeit erfahren.

Kein Aspekt der Familienentwicklung in Deutschland hat damals die familienwissenschaftliche Diskussion in den letzten Jahrzehnten so stark geprägt wie die wahrgenommenen Veränderungen der Familienformen. Obwohl die Diskussion darüber, ob man es bei dieser Entwicklung wirklich mit einer Pluralisierung, also mit dem Auftreten von neuen und somit einer Zunahme der Formen familialen Lebens zu tun hat, schon seit längerem geführt wurde, galt diese Frage als unentschieden. Dementsprechend wurden in den 1990er Jahren zu diesem Thema die meisten Beiträge eingereicht. Dazu kamen weitere Themen wie familiale Generationenbeziehungen und Netzwerke, Instabilität der Ehen, Zusammenhänge zwischen Verfassungsnorm und Lebenswirklichkeit von Familien, aber auch Scheidungsursachen, Familiensituation alter Menschen, Kinderarmut, usw.

Die eingereichten Manuskripte bestätigten, dass die Zeitschrift in der Tat Familenforscher(innen) verschiedener Fachdisziplinen angesprochen hat. Ein Rückblick auf die in der Zeitschrift für Familienforschung in den Jahren 1998 bis 2000 veröffentlichten Beiträge lässt bereits in dieser Phase eine erfreuliche Vielfalt von theoretisch und/oder empirisch behandelten Themen, die in der aktuellen Familienforschung aufgegriffen werden, erkennen. In den Folgejahren wurden der Zeitschrift hauptsächlich Beiträge angeboten, die sich neben den strukturellen Veränderungen von Familien auch mit der sozioökonomischen Lage von Familien, den verwandtschaftlichen Netzwerken, Generationenzusammenhängen und Fragen der Familienpolitik beschäftigten.

Die Familienforschung wurde in diesen Jahren in verschiedenen europäischen Ländern durch jeweils eigene Forschungseinrichtungen betrieben. Deshalb sollten die wichtigsten Institute des europäischen Auslandes durch eingeladene Autoren, die diesen Instituten angehörten, vorgestellt werden. Die Herausgeber hofften, auf diese Weise einen Beitrag zur Vernetzung der für die Familienforschung relevanten Institute vor allem des europäischen Auslandes leisten zu können. Solche Beiträge sind in den Folgejahren u.a. über Forschungseinrichtungen in Österreich, den Niederlanden, Großbritannien und Kanada erschienen.

Im Rückblick fällt allerdings auf, dass in dieser Phase zum Thema Funktionen, Gestaltungsaufgaben und Leistungen von Familien nur selten Beiträge eingereicht wurden. Das gleiche gilt z.B. auch für die Thematisierung der unterschiedlichen familienstrukturellen Bedingungszusammenhänge (z.B. Ein-Eltern-Familie, Zwei-Eltern-Familie oder Nachscheidungsfamilien) der familialen Sozialisation und der Persönlichkeitsentwicklung des Kindes. Auch das Aufwachsen der Kinder in nichtehelichen Lebensgemeinschaften im Vergleich zu auf der Ehe basierenden Familien wird in den angebotenen Beiträgen kaum thematisiert. Dies galt auch für andere Gestaltungsaufgaben der Familie wie z. B. das Konsumverhalten, die wirtschaftliche Produktion (z.B. Heim- oder Telearbeit), die emotionale Unterstützung der Familienmitglieder und familiale Konfliktlosungsstrate- 
gien. Um diesen Trend bewusst zu machen und diesem entgegenzuwirken haben die Herausgeber in regelmäßigen Abständen über den aktuellen Stand der Familienforschung kritisch resümierende Stellungnahmen von namhaften Familienforscherinnen und Familienforschern eingeholt und in der Rubrik „Forum“ veröffentlicht.

Um den wissenschaftlichen Standard der Zeitschrift zu gewährleisten wurden neue, strengere Begutachtungsverfahren für die Veröffentlichung der eingereichten Manuskripte eingeführt. Dazu wurden zunächst zwei oder drei anonymisierte Gutachten eingeholt, in Kenntnis dieser haben die Herausgeber über die Veröffentlichung entschieden. Zur Begutachtung der eingereichten Manuskripte wurden damals vorzugsweise die Mitglieder des Wissenschaftlichen Beirates gebeten. In dieser Phase gehörten dem Wissenschaftlichen Beirat 30 Wissenschaftlerinnen und Wissenschaftler (davon fast jeder Dritte aus verschiedenen europäischen Ländern), die im Bereich der soziologischen, psychologischen, demografischen und pädagogischen Familienforschung als durch eigene Forschungsarbeiten ausgewiesene Experten galten.

Auch als Verlagszeitschrift sollte die Zeitschrift nach Beschluss der Herausgeber und der Redaktion dreimal im Jahr im Umfang von etwa 130 Seiten pro Ausgabe erscheinen.

Wenn unter Berücksichtigung dieser sicherlich anspruchsvollen Ziele ein Zwischenresümee gezogen wird, so kann festgehalten werden, dass durch die in dieser Phase veröffentlichten Artikel die anvisierte Themenvielfalt erheblich gesteigert werden konnte. In den Beiträgen wurden zahlreiche Aspekte der Entwicklung von Familien behandelt, diese Themen boten ein „buntes“ und differenziertes Bild der damaligen „Forschungslandschaft" im Bereich der Familienforschung dar. Was die Beteiligung der verschiedenen Fachdisziplinen angeht, konnte in dieser Phase ein ebenfalls erfreulicher Trend beobachtet werden: Es wurden neben familiensoziologischen Beiträgen immer mehr familienpsychologische, aber auch familienpädagogische und -demografische Themen angeboten. Eine Steigerung der Themenvielfalt erfuhren auch die theoretisch behandelten Themen der Familienforschung.

Die Herausgeber wollten in dieser Phase auch verstärkt dazu beitragen, die interdisziplinäre Kommunikation und Diskussion der verschiedenen, mit Familie und Haushalt befassten Fachdisziplinen (insbesondere Familiensoziologie, -demografie, -psychologie, und -politik, Haushaltswirtschaft, historische Familienforschung) zu fördern. Im Rückblick kann festgestellt werden, dass dies in dieser Phase vielfach gelungen ist; sieht man einmal von der anvisierten Einbeziehung von Haushaltswissenschaft und historischer Familienforschung ab.

Auch die Struktur der Zeitschrift wurde in Verbindung mit der inhaltlichen Neukonzipierung neu festgelegt. festgelegt. Im ersten Teil sollten längere wissenschaftliche Abhandlungen, die den Stand der Forschung mit neuen theoretischen Erkenntnissen und empirischen Befunden bereichern und weiterentwickeln, publiziert werden. In der Rubrik „Forum“ sollten kürzere Diskussionsbeiträge zu kontrovers diskutierten wissenschaftlichen Thesen, zu aktuellen Themen der familienpolitischen Diskussion sowie - wie bereits erwähnt - Selbstdarstellungen von in- und ausländischen Institutionen, die sich der Familienforschung widmeten, veröffentlicht werden.

In der 1998 eingeführten Rubrik „Forschungsnotizen“ sollten kürzere Beiträge, Zwischenergebnisse laufender Projekte aus der Familienforschung, auch Ergebnisse kleinerer Forschungsprojekte, deren Stichproben keine Repräsentativität beanspruchen können, die 
jedoch weiterführende innovative Fragestellungen behandeln und methodischen Kriterien des Faches genügen, veröffentlicht werden.

Unter der geschäftsführenden Herausgeberschaft von Hans-Peter Blossfeld wurde ab dem Jahrgang 2003 eine weitere strukturelle Neuerung eingeführt: Schwerpunktthemenhefte, die eingeladene (Gast-)Herausgeber planen und inhaltlich verantworten sollten. Die anvisierten inhaltlichen Schwerpunkte sollten von kompetenten Wissenschaftler(inne)n konzipiert und vorbereitet werden, wobei sie die Autoren rekrutieren und den jeweiligen Schwerpunkt in einem Einführungstext vorstellen. Später wurde auch die Anzahl der Schwerpunktthemenhefte festgelegt: ab 2008 sollten jährlich zwei Schwerpunkthemenhefte und ein thematisch nicht festgelegtes Heft erscheinen.

Zugleich verfolgten die Herausgeber das Ziel, in unregelmäßigen Abständen beim Verlag Barbara Budrich Sonderhefte im Buchformat zu Themen, die für die Familienforschung besonders aktuell und relevant sind, zu veröffentlichen. In diesen Sonderheften sollten ebenfalls Beiträge aufgenommen werden, die den Gegenstandsbereich des jeweiligen Themas behandeln und einen gegenseitigen Bezug aufweisen. Im Unterschied zu den Schwerpunktthemenheften ist dort die Anzahl der Beiträge wesentlich höher: in ersteren wurden i.d.R. zwischen fünf und sieben Beiträge, in den Sonderheften jedoch im Durchschnitt 14 Beiträge veröffentlicht. Mit der Herausgeberschaft der Sonderhefte sollten in dem jeweiligen Themenbereich besonders ausgewiesene Wissenschaftler(innen) betraut werden.

Dieses Konzept sollte es ermöglichen, einerseits in die aktuelle wissenschaftliche Diskussion durch die zeitnahe Veröffentlichung von Einzelbeiträgen einzugreifen, andererseits aber Forschungsergebnisse in den unterschiedlichen Fachdisziplinen zu einem gemeinsamen Thema der Familienforschung zu bündeln und den aktuellen Forschungsstand widerzuspiegeln und kritisch zu würdigen.

Die Zeitschrift blieb auch in der neuen inhaltlichen Konzeption als Verlagszeitschrift in der Trägerschaft des ifb, das die $Z f F$ weiterhin personell und apparativ unterstützte. Dem ifb wurde zudem vom Verlag vertraglich zugesichert, eigenverantwortlich die Rubrik „ifb-Mitteilungen“ zu gestalten. Den Redakteur der Zeitschrift stellt das ifb: bis August 2001 war dies Hartmut Kasten, seitdem ist es Kurt P. Bierschock. Die Redaktion befand sich bis August 2001 in den Räumen der damaligen ifb-Außenstelle München, seit September 2001 befindet sie sich beim ifb in Bamberg.

Die Zusammensetzung des Herausgebergremiums blieb lange unverändert. 2002 schied Wolfgang Glatzer aus dem Herausgeberkreis aus. Hans-Peter Blossfeld rückte $2003 \mathrm{im}$ Herausgebergremium nach und übernahm als Leiter des Staatsinstituts auch das Amt des Geschäftsführenden Herausgebers. Im Jahre 2008 schied François Höpfinger aus, im gleichen Jahr wurden Johannes Huinink (Universität Bremen) und Michaela Kreyenfeld (Universität Rostock) in dieses Gremium berufen.

Seit 2012 hat Henriette Engelhardt-Wölfler als Leiterin des Staatsinstituts die Position der Geschäftsführenden Herausgeberin inne. Unter ihrer Geschäftsführung kam es zu einem Generationenwechsel in der Herausgeberschaft und einer stärkeren Beteiligung namhafter Familienforscherinnen.

Laszlo A. Vaskovics, Johannes Huinink und Ilona Ostner sind im Jahre 2016, HansPeter Blossfeld ist im vergangenem Jahr (2017) aus dem Herausgeberkreis ausgeschieden. 
Gegenwärtig (2018) gehören dem weiterhin interdisziplinär zusammengesetzten Herausgebergremium an: Henriette Engelhardt-Wölfler (Otto-Friedrich-Universität Bamberg), Anette Eva Fasang (Humboldt-Universität zu Berlin), Michaela Kreyenfeld (Hertie School of Governance Berlin), Birgit Pfau-Effinger (Universität Hamburg), Matthias Pollmann-Schult (Otto-von-Guericke-Universität Magdeburg) und Ulrike Zartler (Universität Wien).

Die veränderte personelle Zusammensetzung hatte natürlich Konsequenzen für inhaltliche Gestaltung, Struktur und „Strategie“ der Zeitschrift. Der neue Herausgeberkreis strebte eine weitere Diversifikation der Beiträge aus verschiedenen familienbezogenen Forschungsbereichen an und hat dies auch realisiert.

Es gab in diesen Jahren auch in der Zusammensetzung des Wissenschaftlichen Beirats der $Z f F$ größere Veränderungen. Insbesondere wurde der Beirat verkleinert und auch verjüngt. Zudem wurde versucht, alle wichtigen Fachdisziplinen der Familienforschung personell einzubinden und die rasche Umsetzung der geplanten stärkeren Internationalisierung der Familienforschung voranzutreiben.

\subsection{Internationalisierung der ZfF/JFR (ab Jahrgang 16, ab 2004)}

Die ersten 15 Jahrgänge richteten sich primär an das Fachpublikum in den deutschsprachigen Ländern. Ab dem 10. Jahrgang (1998) wurde die Zusammenfassung der veröffentlichten Beiträge ins Englische übersetzt. Dies war der erste Schritt in Richtung Öffnung der Zeitschrift für die nicht-deutschsprachigen Leser(innen). Die ZfF sollte aber nach dem Beschluss der Herausgeber auch ein Forum sein für europäisch vergleichende Untersuchungen und Beiträge, die Fragestellungen aus dem Bereich der Familienentwicklung länderübergreifend und ländervergleichend thematisieren. Derartige Beiträge wurden wenn überhaupt - hauptsächlich von Autoren aus nicht-deutschsprachigen europäischen Ländern angeboten. Als zweiter Schritt wurde im Jahre 2005 die deutsche Übersetzung solcher englischsprachigen Beiträge beschlossen. Doch dies stellte die Redaktion und die Herausgeber vor zeitliche, und zum Teil auch finanzielle Probleme. Die Herausgeber hatten bereits im Jahre 2002 in Erwägung gezogen, der europäischen Integration und damit der Europäisierung der Sozialwissenschaften künftig auch bei der Konzeption der Zeitschrift stärker Rechnung zu tragen. Dies sollte unter anderem in Form einer stärkeren Einbeziehung familienwissenschaftlicher Beiträge, auch aus anderen europäischen Ländern geschehen. Als dritter Schritt wurde daher beschlossen, die Zeitschrift, wenn auch behutsam, für Autoren mit nicht-deutscher Muttersprache aus den nicht-deutschsprachigen Ländern Europas zu öffnen. Es wurde beschlossen, in Zukunft mehr Originalbeiträge in englischer Sprache in der Zeitschrift für Familienforschung zu veröffentlichen. Durch die Veröffentlichung von Beiträgen von Wissenschaftler(innen) nicht-deutschsprachiger Länder ist es in den Jahrgängen 15 bis 18 (2001-2004) der Zeitschrift zwar gelungen, Einblicke in die Familienforschung einzelner europäischer Länder exemplarisch zu ermöglichen, aber eben nur exemplarisch.

Die $Z f F$ sollte nach den Bestrebungen der Herausgeber ein herausragendes Publikationsforum für Beiträge aus dem Bereich der europäischen Familienforschung werden. Diesem erklärten Ziel ist die Zeitschrift im Laufe der Folgejahre näher gekommen, doch ein Durchbruch zur Internationalisierung der Zeitschrift ist erst seit 2005 gelungen. Hans- 
Peter Blossfeld hat ab 2003 als Geschäftsführender Herausgeber diese Entwicklung engagiert gefördert. Dieser Wechsel markiert eine weitere Zäsur in der Entwicklung der Zeitschrift und leitete eine neue Phase ein, die ich als die „Internationalisierung der Zeitschrift" bezeichnen möchte. Die Zeitschrift hat sich seit $2005 \mathrm{zu}$ einem zweisprachigen wissenschaftlichen Journal, auch im europäischen Kontext, entwickelt. Nach einem erfolgreich durchlaufenen Begutachtungsprozess werden die Beiträge in deutscher oder in englischer Sprache veröffentlicht, wobei der Titel, die Zusammenfassung und die Schlagwörter auch in der jeweils anderen Sprache abgedruckt werden. Seit 2007 führt die Zeitschrift den Doppeltitel Zeitschrift für Familienforschung/Journal of Family Research. Zu Recht, da die Anzahl englischsprachiger Beiträge in der ZfF/JFR deutlich zunahm und zudem die letzten beiden Sonderhefte 10 (Hrsg.: Zartler/Heintz-Martin/Arránz Becker 2015) und 11 (Hrsg.: Betz/Honig/Ostner) ausschließlich Beiträge in englischer Sprache enthielten.

\subsection{ZfF/JFR online}

Eine weitere Zäsur in der Entwicklung der Zeitschrift ist die Erreichbarkeit der Zeitschrift im Internet, die schrittweise realisiert wurde. Beginnend mit Heft 3/2001 sind die deutschen und englischen Zusammenfassungen aller veröffentlichten Beiträge unter www.journal-of-family-research.eu einsehbar. Des Weiteren stehen dort auch die Volltexte dieser Beiträge bis einschließlich Heft 3/2016 über einem Link zum Social Science Open Access Repository (SSOAR) zur Einsicht und zum Herunterladen zur Verfügung. Gleiches gilt auch für den Internetauftritt der Zeitschrift beim Verlag Barbara Budrich, der unter https://www.budrich-journals.de/index. php/zff zu erreichen ist.

Dem Prinzip des sogenannten moving wall folgend, das besagt, dass zwei Jahre zwischen der Veröffentlichung in gedruckter und digitaler Form und der Freigabe der Volltexte zum kostenfreien Herunterladen liegen müssen, stehen die vollständigen Beiträge ab Heft 1/2017 in der Regel nur den Abonnenten der Druck- bzw. der Druck- und OnlineVersion zur Verfügung.

Darüber hinaus können die einzelnen Beiträge, die ab Heft 1/2017 erschienen sind, gegen Entrichtung einer Gebühr an den Verlag über die bereits erwähnte Zeitschriftenseite des Verlags Barbara Budrich heruntergeladen werden.

Der Verlag bietet zudem den Autor(inn)en der ZfF/JFR im Rahmen seiner Open Express-Option gegen Zahlung einer entsprechenden Gebühr an, ihre Beiträge zeitgleich mit der Veröffentlichung der Druck- und der Online-Ausgabe als open access zu publizieren und somit dem Leser(inn)en kostenlos als PDF zum Herunterladen zur Verfügung zu stellen.

Des Weiteren ist die Zeitschrift in vielen der Online-Zeitschriftenportale enthalten, die die Universitätsbibliotheken ihren Nutzern - zu unterschiedlichen Bedingungen hinsichtlich der Verfügbarkeit der Volltexte - zur Verfügung stellen. Der Zugang erfolgt durch eine so genannte IP-Freischaltung in Form einer digitalen Mehrplatzlizenz oder einer Campuslizenz.

Der Verlag vergibt in Zusammenarbeit mit Crossref für die einzelnen Beiträge der Zeitschrift Digital Object Identifiers, kurz: DOIs. Auf diese Weise können diese eindeutig identifiziert werden, sind weltweit sichtbar und auffindbar. 
Nach Verlagsangaben wurden im Jahre 2014 22.189 Mal Volltexte heruntergeladen, im Verlaufe des gesamten Jahres 2015 wurden 23.783 Zugriffe registriert, im Jahre 2017 war dies bereits 52.051 Mal der Fall. Die letzten Zahlen liegen - aus technischen Gründen - leider nur für den Zeitraum 1. Januar 2018 bis 16. März 2018 vor, in diesen $2 \frac{1}{2} 2$ Monaten wurden 8.817 Zugriffe auf Volltexte registriert.

Neben dem renommierten Social Sciences Citation Index (Web of Science, SSCI, Clarivate Analytics), in welchem die Zeitschrift seit Heft 1/2008 geführt wird, ist die $\mathrm{ZfF} / \mathrm{JFR}$ nach Verlagsangaben gegenwärtig in den folgenden externen Datenbanken vertreten bzw. wird auf den folgenden Webseiten indiziert: CNKI, CNPeReading, Crossref, EBSCO, GBI-Genios, GESIS, Google Scholar, IBR-Online, IBZ-Online, NSD, ProQuest Politics Collection, ProQuest Sociology Collection, ProQuest Social Science Premium Collection, PSYNDEX, PubPsych, scholars-e-library, SCImago, SCOPUS, SSOAR und Ulrichsweb (siehe: https://budrich-journals.de/index.php/zff/index).

\section{Publikationen}

Seit Gründung der Zeitschrift sind rund 90 Hefte, davon 23 Schwerpunktthemenhefte mit inhaltlich unterschiedlich fokussierten Themen erschienen. In den Heften ohne Schwerpunktsetzung und in den so genannten Schwerpunktthemenheften wurden 435 wissenschaftliche Artikel veröffentlicht, davon über 70 englischsprachige Beiträge. Neben den genannten Heften wurden 11 Sonderhefte im Buchform beim Verlag Barbara Budrich zu ausgewählten, klar umrissenen Themen veröffentlicht, davon enthalten die meisten nur deutschund zirka ein Drittel deutsch- und englischsprachige Beiträge. Wie bereits erwähnt, wurden in den letzten zwei Sonderheften nur englischsprachige Artikel veröffentlicht. In diesen elf Sonderheften wurden rund 150 Beiträge publiziert. In allen genannten Heften wurden im Laufe der vergangenen 30 Jahre insgesamt fast 600 Artikel veröffentlicht.

Die in den Schwerpunktthemen- und Sonderheften aufgenommenen Einzelbeiträge sind, wie schon beschrieben, inhaltlich auf das jeweilige Schwerpunkthema fokussiert und aufeinander bezogen. Die Gastherausgeberinnen und -herausgeber resümieren jeweils in ihrer zusammenfassenden Einleitung den wissenschaftlichen Ertrag der einzelnen Beiträge bezüglich aktuellem Forschungstand im ausgewählten Themenbereich.

Diese Publikationen erstrecken sich inhaltlich auf nahezu alle Forschungsgebiete und thematisieren die wichtigsten, empirisch und theoretisch relevanten Fragestellungen der soziologischen, m.E. auch der psychologischen, demografischen, der politikwissenschaftlichen sowie der pädagogischen Familienforschung. Es ist nicht möglich, diese Themenvielfalt der veröffentlichten Beiträge im hier vorgegebenen Rahmen detailliert darzustellen. Ich kann diese hier nur durch die stichwortartige Beschreibung der Themenvielfalt der Schwerpunktthemen- und Sonderhefte exemplarisch illustrieren.

In den ersten Schwerpunktthemenheften wurden folgende Themen fokussiert behandelt: Neuere Entwicklungen im Bereich der Familie (Kinderwunsch und Realität, kinderlose Ehen, Neue Väter). In einem nachfolgenden Schwerpunktthemenheft ging es um die Diskussion der Frage „Wo steht die Familienforschung?“" - dies aus der Sicht der Familiensoziologie, Familienpsychologie und Familienpädagogik. Sowohl der Stand der Theorienbildung in der Familiensoziologie als auch der Stand der empirischen Familiensozio- 
logie wurde in den aufgenommenen Einzelbeiträgen kritisch gewürdigt. Anschließend wurde in einem Schwerpunktthemenheft das Thema „Generationentransfers“ am Beispiel der Unterstützungsleistungen im Alter, Familienpolitik und Müttererwerbstätigkeit unter die Lupe genommen. Durch den Übergang vom Alleinverdienermodell zum Zuverdienerund Doppelverdienermodell ist die wechselseitige Abstimmung von Karrieren von Partnern und Eheleuten in den Mittelpunkt des öffentlichen und wissenschaftlichen Interesses gerückt. Die Herausgeber haben darauf mit der Veröffentlichung eines Schwerpunktthemenheftes zu diesem Thema reagiert. Diese Zusammenhänge wurden auch im Schwerpunktthema „Dual-career couples“ unter Berücksichtigung verschiedener europäischer Länder beschrieben und analysiert. Ein weiteres Heft wurde dem Thema „Kinderarmut in Europa" gewidmet. In einem folgenden Heft wurden die Zusammenhänge zwischen Fertilität und Frauenerwerbstätigkeit anhand empirischer Ergebnisse und Diskussionsbeiträge thematisiert. Auch das höchst aktuelle Thema „Heiratsmuster ethnischer Minderheiten in Europa" wurde in einem Schwerpunktthema fokussiert aufgegriffen. Dasselbe gilt für das Schwerpunktthema „Mobilität und Familie“. Fokussiert wurde auch das Thema der Auswirkungen der gesellschaftlichen und ökonomischen Veränderungen in den Übergangsgesellschaften Mittel- und Osteuropas auf die ,gender relations“. Auch bisher kaum untersuchte Themen wie „Aspekte von Partnerschaftsbörsen“ im Internet wurden als Schwerpunkt thematisiert. Hier ging es um die Prozesse der Partnerwahl auf Online-Kontaktbörsen. Ebenso behandelt wurden Probleme und Fragen der frühen Förderung von Kindern in den Familien. Auch der aktuellen Entwicklung von "Stiefelternfamilien“ wurde ein Schwerpunktthemenheft gewidmet, Dazu kamen in den letzten Jahren Schwerepunktthemen wie die Generationenbeziehungen und Generationsperspektiven in türkischen Familien in Deutschland, Ziele und Wirkungen des Elterngeldes und der Elternzeit, zeitliche Platzierung der Geburt von Kindern und deren Einflussfaktoren, aber auch das komplizierte Verhältnis von Elternhaus, Kind und Schule. In den neueren Veröffentlichungen kamen Schwerpunktthemen wie Kinderlosigkeit und Kinderreichtum in Deutschland, das Spannungsverhältnis zwischen Familienentwicklung und Familienrecht, Väter und Berufstätigkeit und Zeitverwendung in der Familie dazu.

Aktuelle, theoretisch wie politisch bedeutsame Themen der interdisziplinären Familienforschung konnten noch intensiver und umfassender im Rahmen der Sonderhefte analysiert und diskutiert werden. Die bisher veröffentlichten elf Sonderhefte haben folgende Themenbereiche behandelt: Gesellschaftliche Rahmenbedingungen und individuelle Gestaltungsaufgaben der Elternschaft, Eltern-Kind-Beziehungen im Jugend- und früheren Erwachsenenalter. Ein eigenes Sonderheft war den aktuellen Fragen der sozialwissenschaftlichen Männerforschung gewidmet (Das „vernachlässigte“ Geschlecht in der Familienforschung). Das nächste Thema war das komplexe Beziehungsgeflecht zwischen Arbeitszeit, Familienzeit und Lebenszeit. Ein weiteres Heft hat die in der sozialwissenschaftlichen Forschung bekannt gewordenen Prognosen und Szenarien der künftigen Entwicklung der Familie analysiert und diskutiert. Auch damals viel diskutierte Fragen der Partnerschaft und Elternschaft bei gleichgeschlechtlichen Paaren wurde fokussiert behandelt. Die Pluralisierung der Elternschaft und Kindschaft und ihre Konsequenzen für die Gesetzgebung und Rechtsprechung diskutierten Soziologen, Psychologen und Rechtswissenschaftler in einem weiteren Sonderheft. Das höchst aktuelle Thema der Familienund Partnerschaftsentwicklung in Ost- und Westdeutschland im Vergleich wurde eben- 
falls ausführlich behandelt. In den letzten zwei (englischsprachigen) Sonderheften ging es um die Entwicklung von Nachscheidungsfamilien und die Alltagsgestaltung der Elternschaft in europäischen Ländern.

\section{Resümee}

Abschließend möchte ich die beschriebene Entwicklung der ZfF/JFR (z.T. selbst-)kritisch würdigend resümieren. Die erklärte Zielsetzung der Zeitschrift war, die interdisziplinäre Diskussion über alle familienbezogenen Fragestellungen zu fördern, die kritische Auseinandersetzung mit dem aktuellen Forschungsstand zu unterstützen und neue theoretische und methodische Forschungsansätze in der Familienforschung vorzustellen.

Es stellt sich aus der Retrospektive auf 30 Jahre natürlich die Frage: wurden diese Zielsetzungen erreicht?

Die Fragen der Familienforschung stellen sich immer mehr disziplinübergreifend und sind interdisziplinär zu beantworten. Die Entscheidung der Herausgeber, die Zeitschrift für Familienforschung als eine interdisziplinäre Zeitschrift zu konzipieren, hat sich meines Erachtens im Rückblick auf diese 30 Jahre als richtig erwiesen. Die Zielsetzung der Zeitschrift, die interdisziplinäre Diskussion über alle familienbezogenen Fragestellungen zu fördern, die kritische Auseinandersetzung mit dem aktuellen Forschungsstand zu unterstützen und neue Forschungsansätze in der Familienforschung vorzustellen, wurde der Tendenz nach erreicht. Trotz einiger noch nicht oder nur teilweise erreichten, ursprünglich anvisierten Ziele befindet sich die Zeitschrift auf dem richtigen Weg. Sie gilt in Deutschland - und immer mehr auch im europäischen Kontext - inzwischen als ein anerkanntes und geschätztes Forum wissenschaftlicher Publikationen aus dem Bereich der Familienforschung. Sie gilt als die führende wissenschaftliche Zeitschrift für Familienforschung in den Sozial- und Humanwissenschaften in deutschsprachigen und immer mehr auch in anderen europäischen Ländern.

Seit drei Jahrzehnten veröffentlicht die $Z f F / J F R$ Originalarbeiten zu theoretischen, empirischen und methodologischen Fragestellungen über alle Aspekte und Phasen des Zusammenlebens in Familien und Partnerschaften, über den gesamten Lebenslauf sowie zu den Wechselbeziehungen zwischen Familie und Gesellschaft. Die im Laufe der vergangenen drei Jahrzehnte veröffentlichten Beiträge erstrecken sich inhaltlich auf fast alle relevanten und aktuellen Gebiete der soziologischen, und mehrere Gebiete der psychologischen, demografischen, pädagogischen und - wenn auch auf Spezialthemen beschränkt rechtswissenschaftlichen, politikwissenschaftlichen und wirtschaftswissenschaftlichen Familienforschung. Die Autoren sind namhafte Vertreter ihrer Fachrichtungen in Forschung und Lehre, aber vor allem viele jüngere Wissenschaftlerinnen und Wissenschaftler haben ihre familienbezogenen Forschungsergebnisse in der ZfF/JFR publiziert. Man kann resümierend feststellen, dass die Akteure der deutschen und zunehmend auch der europäischen Familienforschung verschiedener Fachdisziplinen die Zeitschrift angenommen haben.

Als richtig hat sich in der Vergangenheit auch die gewählte Struktur der Zeitschrift erwiesen. Sie hat ermöglicht, neben ausführlicheren Beiträgen aus der familienbezogenen Grundlagenforschung kürzere Abhandlungen zu speziellen Forschungsfragen und Kurz- 
berichte über die Zwischen- und Endergebnisse laufender und vor kurzem abgeschlossener Forschungsprojekte regelmäßig zu publizieren.

Besonders möchte ich die Wichtigkeit der Konzipierung von Schwerpunktthemenheften hervorheben. Die regulären Hefte ohne Schwerpunktsetzung haben Autorinnen und Autoren die Möglichkeit gegeben, aktuelle Forschungsergebnisse und Diskussionsbeiträge zeitnah auch jenseits der jeweiligen Schwerpunktthemen zu publizieren. Die Schwerpunktthemenhefte haben wiederum die Möglichkeit eröffnet, zum selben Themenbereich von mehreren Autoren, oft auch aus der Sicht mehrerer Fachdisziplinen Ergebnisse aus der Familienforschung der wissenschaftlichen Öffentlichkeit „verdichtet“ vorzustellen und zur Diskussion zu stellen.

Im Laufe des letzten Jahrzehnts hat sich auch die Struktur der Zeitschrift verändert. Mit den Rubriken „Forum“ und „Forschungsnotizen“ wollten die Herausgeber(innen) es ermöglichen, dass über aktuelle Forschungsprojekte möglichst zeitnah - auch durch kürzere Beiträge - berichtet wird. Insbesondere auch jüngeren Wissenschaftler(inne)n sollte es so ermöglicht werden, Zwischenergebnisse ihrer Forschungsarbeiten zu veröffentlichen. Aufgrund der kontinuierlichen Steigerung der wissenschaftlichen Qualitätskriterien bekamen die regulären Forschungsartikel ein immer größeres Gewicht, sodass das „Forum" entfiel und Forschungsnotizen kaum noch veröffentlicht wurden. Diese Entwicklungen waren $\mathrm{m}$.E. folgerichtig und konsequent.

Auch die Entscheidung von Zeit zu Zeit Sonderhefte zu veröffentlichen, erweist sich aus einer rückblickenden Betrachtung als richtig. Zur inhaltlichen Gestaltung konnten die wichtigsten Autoren des Inlandes und des europäischen Auslandes zusammengeführt werden, die im jeweiligen Themenbereich aktuell forschend tätig waren. Durch die Sonderhefte ist es gelungen den Stand der Familienforschung in den ausgewählten Themenbereichen aktuell abzubilden und zusammenfassend kritisch zu resümieren. Es ist gelungen, Themenbereiche, die bisher in der Familienforschung eher vernachlässigt wurden, in die aktuelle wissenschaftliche Diskussion einzubringen (z.B. Partnerschaft und Elternschaft bei gleichgeschlechtlichen Paaren, Pluralisierung von Kindschaft und Elternschaft, Männerforschung).

Die Entscheidung, die inhaltliche Konzipierung und Herausgabe der Schwerpunktthemenhefte und Sonderhefte an Familienforscherinnen und Familienforschern zu übertragen, die in dem ausgewählten Themenbereich als besonders gut ausgewiesen waren, hat sich als zukunftsweisend für die Zeitschrift erwiesen. Denn es ist ihnen gelungen, im Besitz ihres Überblickswissens einschlägige Beiträge von kompetenten Autoren im Rahmen der betreuten Schwerpunktthemen- und Sonderhefte zu jeweils homogenen Projekten „zusammenzuführen“.

Es war richtig, die Sprachbarrieren für die Verbreitung der Zeitschrift im europäischen Ausland durch Öffnung der Zeitschrift für englischsprachige Beiträge zu überwinden. Dadurch haben die Herausgeber den Weg zur Internationalisierung der Zeitschrift geöffnet.

Sowohl die regulären Hefte als auch die Sonderhefte sind als gedruckte Version und Online erhältlich. Es war richtig, in den letzten Jahren die Online-Version der Zeitschrift auf dem Markt schrittweise durchzusetzen und vorrangig zu fördern, aber zugleich die Printversion (zumindest für eine überschaubare Zeit) beizubehalten.

Als Zielgruppen wurden und werden Forscherinnen und Forscher, die sich mit familienbezogenen Fragestellungen befassen, angesehen: in erster Linie Soziolog(inn)en, Psy- 
cholog(inn)en, und Bevölkerungswissenschaftler(innen), aber auch Politikwissenschaftler(innen), Wirtschaftswissenschaftler(innen), Rechtswissenschaftler(innen), Sozialpädagog(inn)en, Expert(inn)en in der Politikberatung, der Wohlfahrtspflege und in den NGOs und politische Entscheidungsträger(innen). Es gibt aber keine wirklich verlässlichen Informationen darüber, wie sich die Leserschaft der Zeitschrift gegenwärtig zusammensetzt.

Die hier beschriebenen Phasen der Entwicklung haben Herausgeber in wechselnder personeller Zusammensetzung gelenkt und verantwortet. Dies gilt auch für die Geschäftsführung der Herausgeberschaft. Die Entscheidung, die „Amtszeit“ der Herausgeber(innen) zeitlich zu beschränken, war m.E. richtig. Auf diese Weise konnte gewährleistet werden, dass auch die jeweils unterschiedlichen Forschungsschwerpunkte, theoretischen Orientierungen und methodischen Präferenzen der Herausgeber(innen) bei der Gestaltung der Zeitschrift zur Geltung kamen. Es gab fruchtbare Diskussionen unter den Mitgliedern des Herausgeberkreises bei der Festlegung der Inhalte und der Verantwortlichen für die Schwerpunktthemen- und Sonderhefte oder bei der Einladung an bestimmte Autoren, für die Zeitschrift Beiträge einzureichen. Auch die geschäftsführenden Herausgeber haben unterschiedliche inhaltliche wie strategische Ziele gesetzt, wovon die Zeitschrift profitieren konnte.

Und zum Schluss: Ohne die kooperierende, unterstützende Mitwirkung und Begleitung der Verlage Leske + Budrich und seit 15 Jahren des Verlages Barbara Budrich, aber auch ohne das Engagement der Verlage in Belangen der Familienforschung könnte die Zeitschrift derzeit nicht diese positive Bilanz aufweisen. Dafür gebührt dem Verlag Barbara Budrich Respekt und Anerkennung. Anerkennung verdient auch die Redaktion der Zeitschrift für den unermüdlichen Einsatz bei der organisatorischen Begleitung des Begutachtungsprozesses, der redaktionellen Vorbereitung der Beiträge für die Drucklegung, aber auch für die organisatorische Unterstützung des Herausgebergremiums bei der Umsetzung der beschlossenen Maßnahmen und für die erfolgreiche „Brückenfunktion“ bei der oft nicht einfachen Kommunikation zwischen den verschiedenen Akteuren.

Bei der Konzipierung der Zeitschrift war auch daran gedacht, ein Forum auch für die Veröffentlichung der Ergebnisse der Haushaltsforschung, Verwandtschaft- und Lebenslaufforschung anzubieten. Doch auf dem Gebiet der Haushaltsforschung wurden kaum Beiträge angeboten. Dies hat wohl in erster Linie mit dem unbefriedigenden Forschungsstand in diesem Bereich zu tun. Etwas anders verhält es sich bei der familienbezogenen Verwandtschaftsforschung und vor allem bei der Lebenslaufforschung. Aus diesen Bereichen konnten im Laufe der vergangenen drei Jahrzehnte zwar wichtige Beiträge veröffentlicht werden, doch relativ viele und wichtige Ergebnisse der Familienforschung sind in anderen Publikationsorganen ,gelandet“. Dies gilt z.B. für die familienbezogene Lebenslaufforschung, aber auch für die demografische Familienforschung.

Dies hat sicherlich auch mit einer Fehleinschätzung der Zeitschriftenlandschaft zur Zeit der Gründung der Zeitschrift und ihrer Konzipierung als Verlagszeitschrift zu tun. Mit der Festlegung der inhaltlichen Ziele der Zeitschrift war damals eine vermutete Lücke hinsichtlich der auf dem Markt bereits präsenten wissenschaftlichen Zeitschriften anvisiert. Doch die Zeitschrift für Familienforschung musste vom Anfang an mit anderen familienbezogenen Fachzeitschriften und Periodika konkurrieren. Dies gilt insbesondere für Fachzeitschriften im englischen Sprachraum. So muss nach wie vor in Kauf genommen werden, dass Ergebnisse der Familienforschung auch in anderen Fachzeitschriften veröffentlicht werden. 
Dazu kommt, dass insbesondere theoretische Beiträge der soziologischen, psychologischen, sozialpädagogischen und demografischen Familienforschung vorzugsweise in thematisch nicht fokussierten Zeitschriften (Kölner Zeitschrift für Soziologie und Sozialpsychologie, Zeitschrift für Soziologie u.a.) veröffentlicht werden.

Die ursprünglich gehegte Hoffnung, dass sich die Zeitschrift auch zu einem lebendigen Diskussionsforum der interdisziplinären Familienforschung auch zwischen Autoren untereinander und zwischen Autoren und Lesern entwickeln wird, konnte bisher nur teilweise realisiert werden. Die meisten veröffentlichten Beiträge, die in der Erwartung weiterer Diskussionsbeiträge veröffentlicht wurden, sind ohne unmittelbare Resonanz der Leser geblieben.

Und wenn man nun den Blick nach vorne richtet, dann bleibt die m.E. berechtigte Hoffnung, dass es den Herausgeber(inne)n gelingt, nicht nur den erreichten Stand und die Position auf dem deutschen und europäischen Zeitschriftenmarkt zu halten, sondern weiter auszubauen und mit einem eigenen Profil zu bereichern. Der eingeschlagene Weg muss allerdings immer wieder neu überprüft, resümiert und - wenn nötig - korrigiert bzw. modifiziert werden. 